
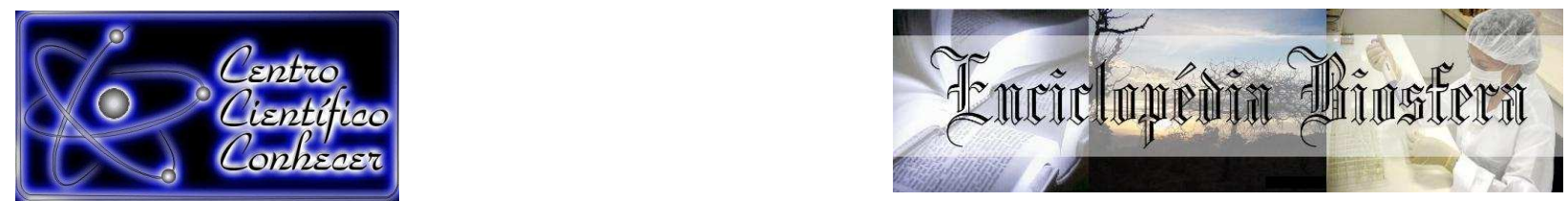

\title{
ESTUDO PRELIMINAR DO TEOR DE METAIS QUANTO A INTEGRIDADE DA AMOSTRA DE SEDIMENTO USANDO PENEIRA E ESPÁTULA DE AÇO INOX
}

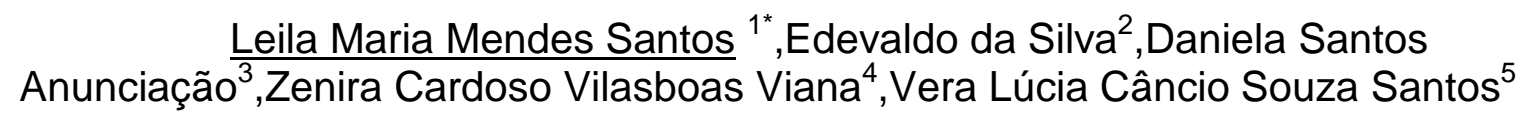

1. Mestre em Geoquímica: Petróleo e Meio Ambiente pela Universidade Federal da Bahia (UFBA). Doutoranda em Química pela UFBA.

2. Doutor em Química pela UFBA. Prof ${ }^{\circ}$ Adjunto III da Universidade Federal de Campina Grande.

3. PhD em Química Analítica UFBA. Prof ${ }^{\mathrm{a}}$ do Instituto de Química e Biotecnologia, Universidade Federal de Alagoas.

4. Doutora em Química pela UFBA. Prof ${ }^{a}$ Associado IV do Departamento de Biofunção do Instituto de Ciências da Saúde da UFBA.

5. PhD em Biogeoquímica pela Universidade de Liverpool. Prof ${ }^{\underline{a}}$ Associado IV do Departamento de Química Analítica da UFBA.

*leilamary_dl@hotmail.com (Rua Barão de Geremoabo, 147 Campus Universitário de Ondina CEP: 40.170-115, Salvador - BA - Brasil)

Recebido em: 08/09/2015 - Aprovado em: 14/11/2015 - Publicado em: 01/12/2015 DOI: http://dx.doi.org/10.18677/Enciclopedia_Biosfera_2015_048

\begin{abstract}
RESUMO
Este trabalho apresenta um estudo comparativo entre os resultados do teor de metais $(\mathrm{Cr}, \mathrm{Cu}, \mathrm{Fe}, \mathrm{Mn}, \mathrm{Mo}, \mathrm{Ni}, \mathrm{Ba}, \mathrm{Sr}, \mathrm{V}$ e $\mathrm{Zn}$ ) presentes em sedimentos manipulados (coleta e peneiramento) com materiais de aço inoxidável e de polietileno. A coleta das amostras foi realizada em outubro de 2011 no manguezal do Rio Passa Vaca-Salvador/Ba. Após a liofilização, pré-tratamento (peneiramento e moagem e digestão com bloco digestor com refluxo), as amostras foram analisadas para a determinação dos elementos por Espectrometria de Emissão Ótica com Plasma Indutivamente Acoplado (ICP OES). Os resultados obtidos indicaram maiores concentrações de metais nas amostras manipuladas com material de aço inoxidável quando comparadas as amostras manuseadas com material de polietileno. Ao ser aplicado o teste de hipóteses nas amostras manipuladas com aço inoxidável observou-se um valor de $p$ abaixo de 0,05 confirmando interferência significativa somente para o $\mathrm{Cr}$ e o $\mathrm{Zn}$, sendo o $\mathrm{Cr}$ considerado como significante e o Zn com extrema significância na análise. O PCA (Principal Component Analysis) realizado, mostrou correlações positivas entre os metais $\mathrm{Ni}, \mathrm{Cr}$, Ba e $\mathrm{Fe}, \mathrm{Zn}$ e $\mathrm{Sr}$ corroborando com as concentrações destes elementos químicos determinados nas amostras de sedimento avaliadas. Além disso, indicou quatro agrupamentos formados em função do local da coleta ilustrado pelo gráfico de escores.
\end{abstract}

PALAVRAS-CHAVE: polietileno, manguezal, ICP OES, PCA. 


\title{
PRELIMINARY STUDY OF CONTENT METAL FOR THE INTEGRITY OF THE SEDIMENT SAMPLE USING SIEVE AND SPATULA STAINLESS STEEL
}

\begin{abstract}
This paper presents a comparative study between the results of the metal content $(\mathrm{Cr}, \mathrm{Cu}, \mathrm{Fe}, \mathrm{Mn}, \mathrm{Mo}, \mathrm{Ni}, \mathrm{Ba}, \mathrm{Sr}, \mathrm{V}$ and $\mathrm{Zn}$ ) present in sediment manipulated (collecting and screening) with stainless steel materials and polyethylene. Sample collection was held in october 2011 in the mangrove of River Passa Vaca, Salvador/Ba. After lyophilization, treatment (screening and grinding) and digestion with digester block with reflux, the samples were analyzed for the determination of elements by Inductively Coupled Plasma Optical Emission Spectrometry (ICP OES). Results showed higher concentrations of metals in the samples handled with stainless steel material when compared to the samples handled polyethylene material. If applying hypothesis testing in the samples handled with stainless steel material, we observed a $p$-value below 0.05 considering significant interference only for $\mathrm{Cr}$ and $\mathrm{Zn}$, being considered significant $\mathrm{Cr}$ and $\mathrm{Zn}$ with extreme significance in the analysis. It was performed PCA (Principal Component Analysis) showing a positive correlation between the metals $\mathrm{Ni}, \mathrm{Cr}$, and $\mathrm{Fe}, \mathrm{Ba}, \mathrm{Sr}$ and $\mathrm{Zn}$ supporting with the concentrations of these chemicals determined in the sediment samples evaluated, besides indicating four groups formed depending on the collection site illustrated by the graph of scores.
\end{abstract}

KEYWORDS: polyethylene, mangrove, ICP OES, PCA.

\section{INTRODUÇÃO}

Manguezais são importantes ecossistemas largamente utilizados em estudos geológicos e biogeoquímicos, os quais fornecem um ambiente com relações de teias alimentares complexas. Esses ecossistemas destacam-se pela riqueza de material orgânico, flora e fauna, contribuindo para manutenção de valores ecológicos e socioeconômicos para populações adjacentes (FARIAS, 2007; SILVA et al., 2013). Essas características, o torna um ecossistema único (LIMA \& TOGNELLA, 2012). Desta forma, suas amostras biológicas e geológicas devem ser criteriosamente coletadas e manipuladas.

Em uma análise química é de fundamental importância, além da seleção adequada do método, as etapas de amostragem, e o tratamento, onde as amostras deverão ser devidamente processadas antes da determinação propriamente dita dos analitos, a fim de garantir a representatividade das amostras.

$\mathrm{Na}$ maioria das vezes os ambientes a serem amostrados encontram-se bastante impactados, possuindo materiais grosseiros associados a amostras de sedimentos e biota, sendo necessário tratamento prévio destas amostras as etapas de amostragem, extração e determinação de espécies químicas. Em alguns trabalhos que envolvem análises biogeoquímicas, é comum a utilização de materiais como espátulas, peneiras, aparelhos de moagem e trituramento inadequados, comprometendo a segurança dos resultados.

Segundo NOGUEIRA (2003), um correto procedimento de coleta e preparação de amostras geológicas e biológicas oferece uma avaliação eficiente e segura nos dados obtidos de concentrações das espécies químicas avaliadas. É essencial que etapas de amostragem, preparação e solubilização destas amostras sejam realizadas de modo satisfatório e adequadas, pois erros significativos podem ser introduzidos durante estas etapas. 
Um número limitado de estudos aborda a contaminação pelo material utilizado durante a coleta, tratamento e armazenamento das amostras. É denominado de contaminação primária, o primeiro contato com a superfície de materiais que podem adicionar teores do analito que será determinado nas análises requeridas. No geral a maioria dos estudos enfatiza a contaminação por outras amostras (contaminação cruzada), que se encontram nas superfícies dos materiais adversos e equipamentos. Comumente estas amostras foram anteriormente manipuladas e os materiais ou equipamentos não foram descontaminados de forma correta e eficiente (SERTEK, 2010).

Pesquisas pioneiras realizadas por THOMPSON \& BANKSTON (1970) em Massachusetts, afirmaram que houve adição de concentrações de $\mathrm{Fe}, \mathrm{Zn}, \mathrm{Ni}$, Co e Mn pelo uso de peneiras de aço inoxidável, durante a manipulação de amostras de solo.

Em estudos mais recentes, SERTEK (2010), publicou que material de aço inoxidável utilizado na redução granulométrica de amostras geológicas contribuiu para a contaminação de $\mathrm{Fe}, \mathrm{Cr}$ e $\mathrm{Mn}$ em maiores concentrações e de $\mathrm{Cu}, \mathrm{Ni}, \mathrm{Zn}, \mathrm{W}$, $\mathrm{Nb}$, Mo, Sc em menores concentrações. Neste estudo britadores, moinhos e prensas de aço foram utilizados para trituração de amostras geológicas.

O presente trabalho tem o proposito de averiguar se há influencia, do material (aço ou polietileno) utilizado durante a amostragem e peneiramentos, nos resultados de analise de uma amostra.

\section{MATERIAL E MÉTODOS}

A amostragem foi realizada em 14 de outubro de 2011 em estação seca, na maré baixa (CETESB, 2011). Constou de uma coleta sistemática de sedimentos superficiais $(0,0$ a $5,0 \mathrm{~cm})$ do substrato do manguezal do rio Passa Vaca, localizado a 12ำ 57'37'S e 3824'06"W, na região próxima a foz do rio Jaguaribe.

Foram definidos quatro pontos de amostragem (Figura 1), coletando-se oito amostras (cerca de $50 \mathrm{~g}$ cada) de sedimentos superficiais do manguezal. Metade delas utilizando espátula de polietileno e a outra metade utilizando espátula de aço inox. Cada tipo de espátula previamente descontaminada foi ambientada nas águas da beira do rio. Este procedimento foi realizado, colhendo-se as amostras lado a lado, dentro de um quadrado com aproximadamente $25 \times 25 \mathrm{~cm}$, procurando-se estabelecer uma distancia de aproximadamente $80 \mathrm{~m}$ de um ponto ao outro, tendo como ponto inicial o estuário (ALMEIDA, 2009).

As coordenadas geográficas dos pontos amostrais foram obtidas através de um GPS (Global Positioning System) e estão indicadas na Tabela 1. Após a coleta, cada amostra foi acondicionada em sacos de polietileno, identificada e transportada em uma caixa térmica com gelo, para sua conservação. Em seguida as amostras foram levadas ao laboratório do NEA (Núcleo de Estudos Ambientais) onde foram armazenadas sob refrigeração, para preservação dessas amostras até a análise laboratorial. 


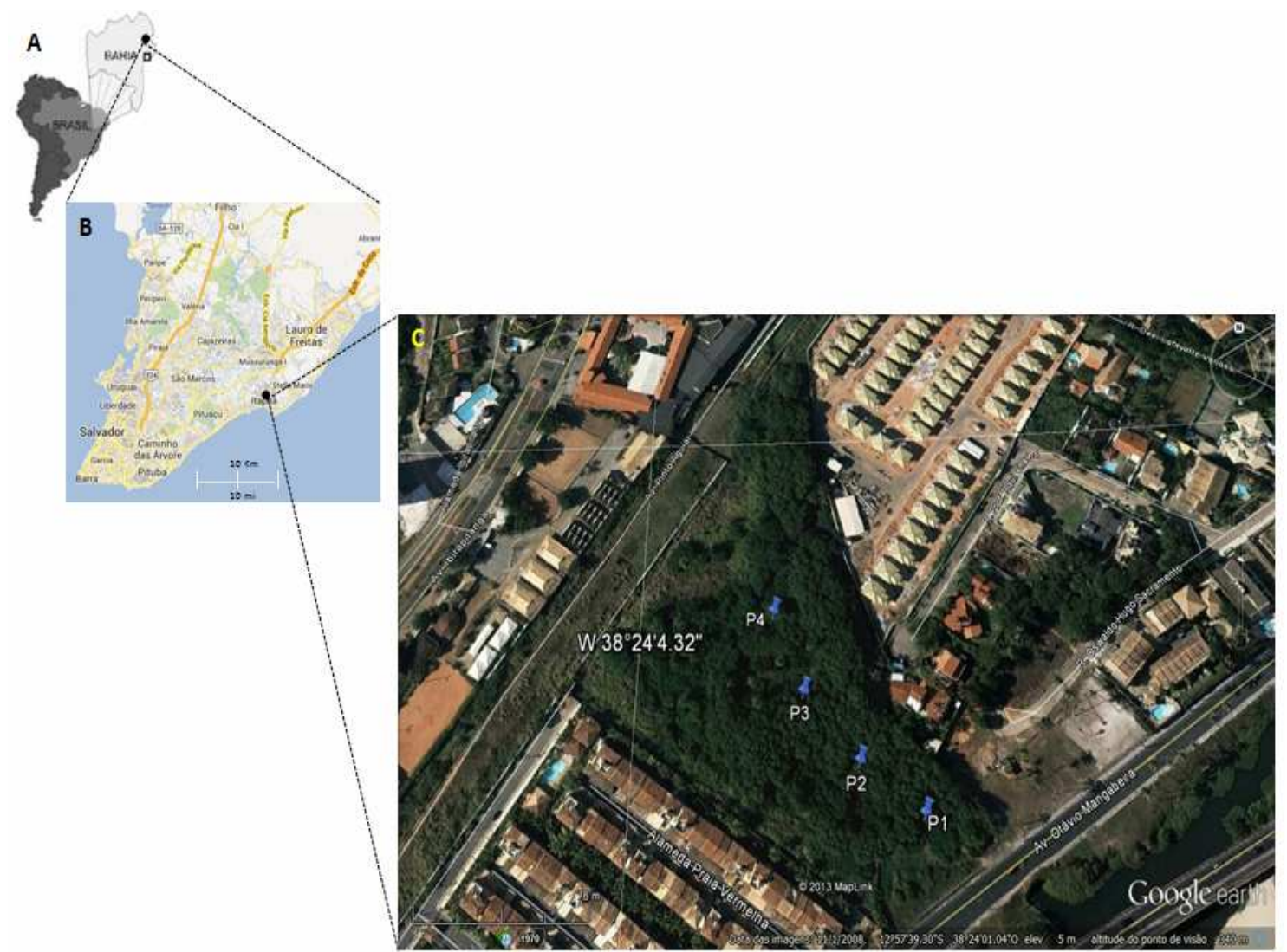

FIGURA 1. Área de estudo, estuário do rio Passa Vaca, situado na Avenida Otávio Mangabeira, Bairro de Patamares, Salvador-BA: A- América Latina, Brasil, Bahia. B- Cidade de Salvador, Ba. C- Pontos de amostragens.

TABELA 1 - Coordenadas Geográficas dos pontos amostrais no manguezal do rio Passa Vaca, Salvador, Bahia.

\begin{tabular}{cc}
\hline Amostras & Coordenadas geográficas \\
\hline $\mathrm{S}_{1}: \mathrm{P}_{1}$ e $\mathrm{A}_{1}$ & $12^{\circ} 57^{\prime} 41.9286^{\prime \prime} \mathrm{S} 38^{\circ} 23^{\prime} 59.7948^{\prime \prime} \mathrm{W}$ \\
$\mathrm{S}_{2}: \mathrm{P}_{2}$ e A $A_{2}$ & $12^{\circ} 57^{\prime} 41.2014^{\prime \prime} \mathrm{S} 38^{\circ} 24^{\prime} 00.7410^{\prime \prime} \mathrm{W}$ \\
$\mathrm{S}_{3}: \mathrm{P}_{3}$ e $A_{3}$ & $12^{\circ} 57^{\prime} 40.1976 " \mathrm{~S} 38^{\circ} 24^{\prime} 01.5906^{\prime \prime} \mathrm{W}$ \\
$\mathrm{S}_{4}: \mathrm{P}_{4}$ e $A_{4}$ & $12^{\circ} 57^{\prime} 38.9736^{\prime \prime} \mathrm{S} 38^{\circ} 24^{\prime} 02.0658^{\prime \prime} \mathrm{W}$ \\
\hline
\end{tabular}

S: Estação de amostragem; P: amostras manipuladas com material de polietileno; A: amostras manipuladas com material de aço inoxidável.

\section{Preparo das amostras}

As amostras foram liofilizadas (liofilizador de marca TERRONI FAUVEL e modelo LT6000), tamisadas a $2 \mathrm{~mm}$ (para a retirada de todo o material grosseiro como pedaços de madeira, pequenos crustáceos e outros utilizando peneiras aço inoxidável ou de polietileno), quarteadas e digeridas em bloco digestor com refluxo para mineralização, conforme o método desenvolvido por LUTZ \& THOMPSON (2012).

Para o preparo das amostras e da solução padrão foi utilizada água ultra pura obtida de um sistema de purificação MILLI-Q ${ }^{\circledR}$ Pluswater (Millipore Molsheim, França). Todos os materiais utilizados foram previamente lavados com água e colocados em solução de detergente por 24 horas. Em seguida, após enxague com água destilada, foi imersa em solução de $\mathrm{HNO}_{3}$ (10\%) pelo mesmo período e, 
subseqüentemente, lavados três vezes com água destilada seguida de lavagem com água MILLI-Q ${ }^{\circledR}$ (resistividade de $18.2 \mathrm{M} \Omega \mathrm{cm}^{-1}$ ) e armazenados em recipientes até análises. Todos os solventes e reagentes utilizados foram de grau de analítico. Para dissolução da amostra foi usado $\mathrm{HCl}\left(37 \%\right.$ Merck) e $\mathrm{HNO}_{3}$ ultrapuro $\left(65 \% \mathrm{~m} \mathrm{~m}^{-1}\right)$ e $\mathrm{H}_{2} \mathrm{O}_{2}$ (30\% Merck, Alemanha).

As amostras foram mineralizadas, por digestão parcial, utilizando o método de LUTZ \& THOMPSON (2012), que é baseado no método 3050B EPA cujo fluxograma está ilustrado na Figura 2. A digestão consistiu na pesagem em balança analítica, das amostras (aproximadamente $0,5 \mathrm{~g}$ ), em tubos digestores de vidro $(250 \mathrm{~mm} \times 50$ $\mathrm{mm}$ ). Nos quais, no total, $5,0 \mathrm{~mL}$ de $\mathrm{HNO}_{3} 14,4 \mathrm{~mol} \mathrm{~L}^{-1}$ e $3,0 \mathrm{~mL}$ de $\mathrm{H}_{2} \mathrm{O}_{2} 30 \%\left(\mathrm{v} \mathrm{v}^{-1}\right)$, foram adicionados e aquecidos em bloco digestor, utilizando "dedos frios" para evitar perda por evaporação e causar o efeito do refluxo. Neste aparato é colocada água fria para resfriar a parte superior do tubo digestor causando refluxo e condensação das espécies voláteis (FEREIRA et al., 2012). O tempo utilizado na digestão foi 30 minutos à $120^{\circ} \mathrm{C}$ e 1,5 horas à $95^{\circ} \mathrm{C}$. Em seguida, foram adicionados $5,0 \mathrm{~mL}$ de $\mathrm{HCl}$ $12 \mathrm{~mol} \mathrm{~L}^{-1}$. Após resfriamento em temperatura ambiente, a solução foi transferida para tubos de centrífuga de polietileno (Falcon) e o volume foi ajustado para $50 \mathrm{~mL}$ com água MILLI-Q ${ }^{\circledR}$.

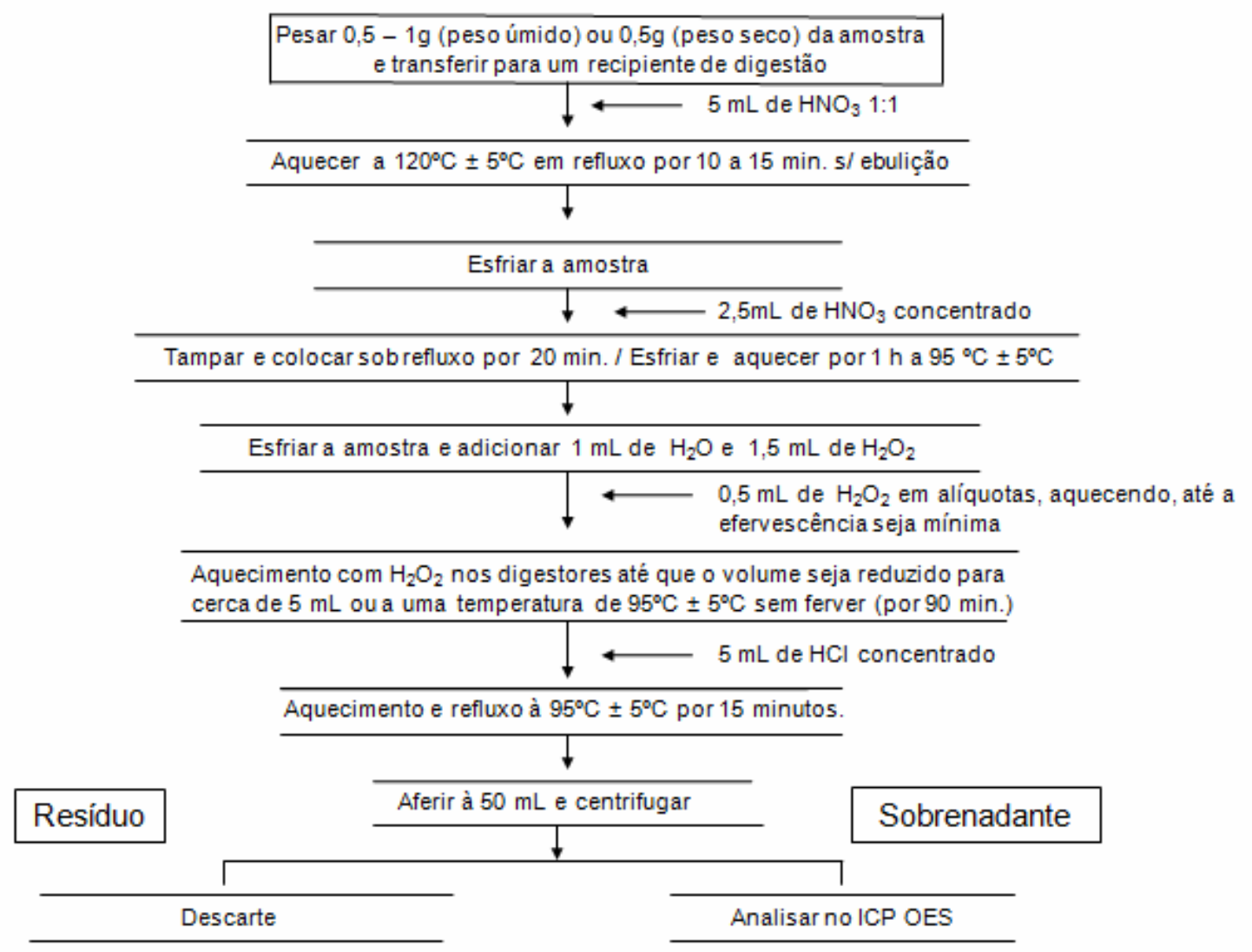

FIGURA 2. Fluxograma referente ao método LUTZ e THOMPSON (2012) de extração de metais.

\section{Determinação dos elementos}

Os elementos foram determinados por Espectrometria de Emissão Ótica com Plasma Indutivamente Acoplado (ICP OES) simultâneo com visão axial, cujas condições estão descritas na Tabela 2. 
Para as confecções das tabelas de dados dos metais determinados pelo ICP OES foram utilizadas as linhas de emissão mais sensíveis de acordo com equipamento. São elas: bário (Ba: 455.403,II); cromo (Cr: 267.716,II); cobre (Cu: 237.395,I); ferro (Fe: 238.204,II); manganês (Mn: 257.610,II); molibidênio (Mo: 202.032,); níquel (Ni: 231.604,II); estrôncio (Sr: 407.771,I); vanádio (V: 292.401,II); zinco (Zn: 213.857,I), no qual "I" corresponde a linha de emissão atômica e "II" corresponde a linha de emissão iônica

Todas as análises das amostras foram realizadas em triplicatas. As soluções estoque utilizadas para preparo das soluções padrão foram de alto grau de pureza e monoelementar (Merck - Darmstadt, Alemanha). Uma solução multielementar foi preparada para determinação de elementos traço com concentração de $50 \mathrm{mg} \mathrm{L}^{-1}$. A curva analítica de calibração foi feita pela diluição da solução multielementar em cada ponto da curva, para isto foram preparados nove padrões numa faixa de concentração de 0,05 a $5 \mathrm{mg} \mathrm{L}^{-1}$.

TABELA 2. Parâmetros do equipamento ICP OES empregado para determinação dos elementos nas amostras de sedimentos coletadas no manguezal do rio Passa Vaca, Salvador, Bahia.

\begin{tabular}{lc}
\hline \multicolumn{1}{c}{ Parâmetros } & Valores \\
\hline Potência RF $(\mathrm{kW})$ & 1,3 \\
Vazão do gás de nebulização $\left(\mathrm{L} \mathrm{min}{ }^{-1}\right)$ & 0,70 \\
Vazão do gás auxiliar $\left(\mathrm{L} \mathrm{min}^{-1}\right)$ & 1,5 \\
Vazão do gás do plasma $\left(\mathrm{L} \mathrm{min}^{-1}\right)$ & 15 \\
Tempo de integração (s) & 1,0 \\
Tempo de estabilização (s) & 15 \\
Tempo de leitura (min) & 1 \\
Replicatas & 3 \\
Nebulizador & Concêntrico \\
Câmara de Nebulização & Ciclônica \\
\hline
\end{tabular}

Os brancos e as soluções padrão de calibração foram analisados seguindo a mesma metodologia das amostras. Para cada batelada de amostras, no bloco digestor, foram adicionados três brancos preparados com o mesmo processo de digestão.

A validação do método foi realizada por meio de análises do material de referência NIST Standard Reference Material 1646a (Estuarine Sediment) introduzidos nas análises aleatoriamente e tendo o mesmo tratamento que as amostras digeridas. A exatidão do método foi determinada em termos percentuais do coeficiente de variação a partir dos valores de referência reportados para o material certificado (CRM). Enquanto a precisão do método foi determinada com a avaliação dos valores médios de Desvio Padrão Relativo (RSD) calculados pela triplicata, considerando-se estes aceitáveis sendo inferior a 15\%. Os limites de detecção (LOD) e quantificação (LOQ), para cada analito, foram calculados considerando a concentração que corresponde a 3 e 10 vezes, respectivamente, o desvio padrão (s) de 10 medidas independentes do branco (medidas de intensidade para o ICP OES) dividido pelo seu coeficiente da curva de calibração (S) (TEMPLETON et al., 2000). 


\section{Análises Estatísticas}

O teste de hipóteses foi realizado no programa GraphPad Software Instat, avaliando-se o nível de significância entre as concentrações obtidas nos dois materiais empregados.

Análises estatísticas foram realizadas através de Software Statística versão 8, avaliando-se valores de coeficientes da correlação de Spearman e Análise de Componentes Principais.

\section{RESULTADOS E DISCUSSÕES}

Os valores médios e respectivos desvios padrão das concentrações dos metais encontrados nas amostras de sedimentos coletados encontram-se discriminados na Tabela 3. Foram obtidas maiores concentrações destas espécies metálicas das amostras coletadas e manipuladas com espátulas e peneiras de aço inoxidável. Com destaque dos altos teores de $\mathrm{Mn}, \mathrm{V}$ e $\mathrm{Zn}$.

$\mathrm{Na}$ Tabela 4 estão apresentados os valores de $p$ encontrados, para a avaliação de cada material utilizado, a fim de verificar se os dados são compatíveis com hipótese de que não há interferência do material utilizado sobre a determinação das espécies químicas estudadas.

De acordo com a literatura, quando o valor de $p$ é menor que 0,05 , há a obtenção de resultados significativos, tendo que aceitar a Hipótese Nula $\left(\mathrm{H}_{0}\right.$ : hipótese que traduz a ausência do efeito que se quer verificar). Por outro lado para valores acima de 0,05 ( $\not \square 0,05)$, resultam em dados obtidos não significativos, podendo-se a assim rejeitar a $\mathrm{H}_{0}$ e adotar a Hipótese Alternativa (que quer se verificar), pois há 5 chances de 100 de que a Hipótese Nula esteja correta, possuindo assim $95 \%$ de confiança. Alguns autores consideram valores de $p$ menores que 0,01 muito significativos (TEÓFILO \& FERREIRA, 2006).

Conforme os resultados obtidos no teste de hipóteses, os elementos $\mathrm{Cr}$ e $\mathrm{Zn}$ foram considerados significantes e de extrema significância, respectivamente, pois 0 Cr apresentou-se significante em $50 \%$ das amostras investigadas e o $\mathrm{Zn}$ em todas elas, levando a conclusão de que o material de aço inoxidável interferiu significativamente na análise destes metais, sugerindo a não utilização destes materiais em análises similares e que possivelmente estes metais estão presentes no material utilizado. 
TABELA 3. Médias e desvios padrão das concentrações dos elementos químicos investigados $\mathrm{Fe}\left(\mu \mathrm{g} \mathrm{g} \mathrm{g}^{-1} \times 10000\right)$ e $\mathrm{Cr}$, Cu, Mn, $\mathrm{Zn}, \mathrm{Sr}, \mathrm{Ba}, \mathrm{Ni}, \mathrm{V}$, Mo $\left(\mu \mathrm{g} \mathrm{g}^{-1}\right)$ nos quatro pontos de coleta de amostras de sedimento de manguezal do rio Passa Vaca, Salvador, Bahia.

\begin{tabular}{ccccccccc}
\hline & \multicolumn{3}{c}{$\mathbf{S}_{\mathbf{1}}$} & & $\mathbf{S}_{\mathbf{2}}$ & & $\mathbf{S}_{\mathbf{3}}$ & $\mathbf{A}$ \\
\cline { 2 - 8 } & $\mathbf{P}$ & $\mathbf{A}$ & $\mathbf{P}$ & $\mathbf{A}$ & $\mathbf{P}$ & $\mathbf{A}$ & $\mathbf{P}$ & $\mathbf{A}$ \\
\hline $\mathrm{Cr}$ & $7,70 \pm 0,65$ & $10,85 \pm 0,65$ & $28,28 \pm 0,95$ & $36,26 \pm 2,59$ & $31,48 \pm 1,07$ & $35,63 \pm 1,39$ & $53,09 \pm 0,51$ & $60,88 \pm 0,12$ \\
$\mathrm{Cu}$ & $6,63 \pm 0,16$ & $9,09 \pm 1,59$ & $26,67 \pm 1,57$ & $29,91 \pm 2,61$ & $16,44 \pm 0,26$ & $19,82 \pm 1,18$ & $23,70 \pm 1,30$ & $34,03 \pm 0,47$ \\
$\mathrm{Fe}$ & $0,40 \pm 0,02$ & $0,57 \pm 0,03$ & $0,19 \pm 0,07$ & $0,23 \pm 0,11$ & $0,20 \pm 0,24$ & $0,21 \pm 0,05$ & $0,28 \pm 0,07$ & $0,35 \pm 0,03$ \\
$\mathrm{Mn}$ & $10,84 \pm 0,15$ & $14,65 \pm 0,85$ & $27,36 \pm 1,26$ & $36,18 \pm 0,94$ & $22,93 \pm 0,53$ & $23,86 \pm 0,50$ & $60,57 \pm 2,67$ & $105,71 \pm 1,23$ \\
$\mathrm{Zn}$ & $8,30 \pm 0,48$ & $15,12 \pm 1,73$ & $47,95 \pm 2,66$ & $51,53 \pm 0,61$ & $17,04 \pm 0,20$ & $22,17 \pm 2,10$ & $37,21 \pm 0,94$ & $51,54 \pm] 0,35$ \\
$\mathrm{Sr}$ & $11,77 \pm 1,50$ & $12,97 \pm 1,06$ & $27,39 \pm 3,04$ & $35,84 \pm 1,82$ & $15,41 \pm 0,54$ & $17,90 \pm 0,55$ & $26,24 \pm 0,54$ & $35,80 \pm 1,06$ \\
$\mathrm{Ba}$ & $5,48 \pm 0,16$ & $6,73 \pm 0,12$ & $19,960,79$ & $22,00 \pm 1,95$ & $13,81 \pm 1,05$ & $15,87 \pm 0,26$ & $23,19 \pm 1,03$ & $34,49 \pm 1,39$ \\
$\mathrm{Ni}$ & $1,42 \pm 0,25$ & $1,43 \pm 0,01$ & $4,12 \pm 0,11$ & $4,49 \pm 0,05$ & $3,34 \pm 0,41$ & $4,45 \pm 0,39$ & $5,84 \pm 0,57$ & $8,00 \pm 0,20$ \\
$\mathrm{~V}$ & $8,54 \pm 0,49$ & $13,09 \pm 0,75$ & $36,54 \pm 2,03$ & $41,83 \pm 3,40$ & $37,54 \pm 0,52$ & $43,46 \pm 1,93$ & $62,96 \pm 1,92$ & $73,14 \pm 0,34$ \\
$\mathrm{Mo}$ & $\square \mathrm{LoQ}$ & $0,67 \pm 0,03$ & $1,23 \pm 0,07$ & $1,55 \pm 0,33$ & $0,74 \pm 0,01$ & $0,87 \pm 0,27$ & $0,71 \pm 0,14$ & $0,46 \pm 0,21$ \\
\hline
\end{tabular}

S: Estação de Coleta; P: Polietileno; A: Aço inoxidável. 
TABELA 4. Médias ( $\left.\mu \mathrm{g} \mathrm{g}^{-1}\right)$ das concentrações, desvios padrão e valores de $p$ dos elementos químicos determinados das amostras de sedimento do manguezal do rio Passa Vaca, Salvador, Bahia.

\begin{tabular}{|c|c|c|c|}
\hline Elemento & $\mathbf{P}$ & A & $p$ value \\
\hline \multirow{4}{*}{$\mathrm{Cr}$} & S1: $7.70 \pm 0.65$ & $S 1: 10.85 \pm 0.65$ & $0.2877 \mathrm{~ns}$ \\
\hline & S2: $28.28 \pm 0.95$ & $S 2: 36.26 \pm 2.59$ & $0.5169 \mathrm{~ns}$ \\
\hline & S3: $31.48 \pm 1.07$ & S3: $35.63 \pm 1.39$ & $0.0193 \mathrm{~s}$ \\
\hline & $S 4: 53.09 \pm 0.51$ & $S 4: 60.88 \pm 0.12$ & $0.0189 \mathrm{~s}$ \\
\hline \multirow{4}{*}{$\mathrm{Cu}$} & S1: $6.53 \pm 0.16$ & S1: $9.09 \pm 0.59$ & $0.1428 \mathrm{~ns}$ \\
\hline & S2: $26.67 \pm 1,57$ & S2: $29.91 \pm 2.61$ & $0.6855 \mathrm{~ns}$ \\
\hline & S3: $16.44 \pm 0.26$ & S3: $19,88 \pm 1,18$ & $0.1903 \mathrm{~ns}$ \\
\hline & S4: $27,70 \pm 1,30$ & S4: $34.03 \pm 0.47$ & $0.6001 \mathrm{~ns}$ \\
\hline \multirow{4}{*}{$\mathrm{Fe}$} & S1: $3961.41 \pm 191.26$ & S1: $5734,74 \pm 284,74$ & $0.2302 \mathrm{~ns}$ \\
\hline & S2: $19181,59 \pm 674.39$ & S2: $23034,30 \pm 1183,03$ & $0.9519 \mathrm{~ns}$ \\
\hline & S3: $19882,12 \pm 237,06$ & S3: $21728.06 \pm 545.89$ & $0.0653 \mathrm{~ns}$ \\
\hline & S4: $28101.65 \pm 727,27$ & S4: $35552,34 \pm 260,13$ & $0.5622 \mathrm{~ns}$ \\
\hline \multirow{4}{*}{$\mathrm{Ni}$} & S1: $60,57 \pm 2,67$ & $S 1: 0,46 \pm 0,21$ & $0.6642 \mathrm{~ns}$ \\
\hline & S2: $4,12 \pm 0,11$ & S2: 4,49 $\pm 0,05$ & $0.5668 \mathrm{~ns}$ \\
\hline & S3: $3,34 \pm 0,41$ & S3: $4,45 \pm 0,39$ & $0.3223 \mathrm{~ns}$ \\
\hline & S4: $5,84 \pm 0,57$ & S4: $8,00 \pm 0,20$ & $0.0263 \mathrm{~s}$ \\
\hline \multirow{4}{*}{$\mathrm{Ba}$} & $S 1: 5,48 \pm 0,16$ & $\mathrm{~S} 1: 6,73 \pm 0,12$ & $0.1473 \mathrm{~ns}$ \\
\hline & S2: $19,96 \pm 0,79$ & S2: $22,00 \pm 1,95$ & $0.2531 \mathrm{~ns}$ \\
\hline & S3: $13,81 \pm 1,05$ & S3: $15,87 \pm 0,26$ & $0.9406 \mathrm{~ns}$ \\
\hline & $S 4: 23,19 \pm 1,03$ & S4: $34,49 \pm 1,39$ & $0.0018 \mathrm{~s}$ \\
\hline \multirow{4}{*}{$\mathrm{Sr}$} & S1: $11,77 \pm 1,50$ & S1: $12,97 \pm 1,06$ & $0.6964 \mathrm{~ns}$ \\
\hline & S2: $35,84 \pm 1,82$ & S2: $27,39 \pm 3,04$ & $0.7828 \mathrm{~ns}$ \\
\hline & S3: $15,41 \pm 0,54$ & S3: $17,90 \pm 0,55$ & $0.8359 \mathrm{~ns}$ \\
\hline & S4: $26,24 \pm 0,54$ & $S 4: 35,80 \pm 1,06$ & $0.4958 \mathrm{~ns}$ \\
\hline \multirow{4}{*}{ V } & S1: $8,54 \pm 0,49$ & S1: $13,09 \pm 0,75$ & $0.2117 \mathrm{~ns}$ \\
\hline & S2: $36,54 \pm 2,03$ & S2: $41,83 \pm 3,40$ & $0.8530 \mathrm{~ns}$ \\
\hline & S3: $37,54 \pm 0,52$ & S3: $43,46 \pm 1,93$ & $0.0439 \mathrm{~s}$ \\
\hline & S4: $62,96 \pm 1,92$ & S4: $73,14 \pm 0,34$ & $0.2891 \mathrm{~ns}$ \\
\hline \multirow{4}{*}{$\mathrm{Zn}$} & $\mathrm{S} 1: 8,30 \pm 0,48$ & S1: $15,12 \pm 1,73$ & $<0.0001$ es \\
\hline & 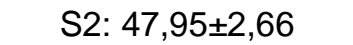 & S2: $51,53 \pm 4,61$ & 0.0005 es \\
\hline & S3: $17,04 \pm 1,20$ & S3: $22,17 \pm 2,10$ & 0.0009 es \\
\hline & S4: $37,21 \pm 0,94$ & S4: $51,54 \pm 0,35$ & $<0.0001$ es \\
\hline \multirow{4}{*}{$\mathrm{Mn}$} & S1: $10,84 \pm 0,15$ & S1: $14,65 \pm 0,85$ & $0.0318 \mathrm{~s}$ \\
\hline & S2: $27,36 \pm 1,26$ & S2: $36.18 \pm 2.94$ & $0.4729 \mathrm{~ns}$ \\
\hline & S3: $22.93 \pm 0.53$ & S3: $23.86 \pm 0.50$ & $0.0548 \mathrm{~ns}$ \\
\hline & $S 4: 60.57 \pm 2.67$ & S4: $105.71 \pm 1.23$ & $<0.0001$ es \\
\hline \multirow{4}{*}{ Mo } & $\mathrm{S} 1:<\mathrm{LOQ}$ & S1: $0,67 \pm 0,03$ & $0.4031 \mathrm{~ns}$ \\
\hline & S2: $1,55 \pm 0,33$ & S2: $1,23 \pm 0,07$ & $0.7058 \mathrm{~ns}$ \\
\hline & S3: $0,74 \pm 0,01$ & S3: $0,87 \pm 0,27$ & $0.4393 \mathrm{~ns}$ \\
\hline & S4: $60,57 \pm 2,67$ & $S 4: 0,46 \pm 0,21$ & $0.6642 \mathrm{~ns}$ \\
\hline
\end{tabular}

S: Estação de Coleta; P: Polietileno; A: Aço inoxidável.

O p-valor foi classificado como ns: não significante; s: significante; es: extremamente significante.

Os valores foram extremamente significativos a $\mathrm{p} 0,01$, para o $\mathrm{Zn}$ e significativos a p0,05 para o $\mathrm{Cr}$. Para identificar grupos de elementos, com comportamento similar e o comportamento geral dos dados, foi utilizado o gráfico da projeção das variáveis e das amostras, explorando a primeira e a segunda componentes principais numa PCA. 
Objetivando avaliar as possíveis relações entre os teores dos metais encontrados nos sedimentos, foram calculadas as correlações de Spearman, considerando um nível de significância p0,01 e p0,05. Na Tabela 5, são indicados coeficientes de correlação de Spearman, no qual se observa forte correlação positiva entre os elementos $\mathrm{Fe}, \mathrm{Ni}, \mathrm{Cr}$, Ba e $\mathrm{V}$, enquanto destaca-se valores de coeficiente negativo indicando a fraca correlação negativa entre os metais Mn e Mo. Este último elemento apresenta ínfima correlação com todos os outros metais estudados.

TABELA 5. Matriz de correlação entre as concentrações de elementos químicos encontrados nas amostras de sedimento coletadas no manguezal do rio Passa Vaca, Salvador, Bahia.

\begin{tabular}{lllllllllll}
\hline & $\mathrm{Cr}$ & $\mathrm{Cu}$ & $\mathrm{Fe}$ & $\mathrm{Mn}$ & $\mathrm{Mo}$ & $\mathrm{Ni}$ & $\mathrm{Ba}$ & $\mathrm{Sr}$ & $\mathrm{V}$ & $\mathrm{Zn}$ \\
\hline $\mathrm{Cr}$ & 1,00 & & & & & & & & & \\
$\mathrm{Cu}$ & $\mathbf{0 , 8 9}$ & 1,00 & & & & & & & & \\
$\mathrm{Fe}$ & $\mathbf{0 , 9 9}$ & $\mathbf{0 , 9 3}$ & 1,00 & & & & & & \\
$\mathrm{Mn}$ & $\mathbf{0 , 8 9}$ & $\mathbf{0 , 7 9}$ & 0,85 & 1,00 & & & & & & \\
$\mathrm{Mo}$ & 0,21 & 0,50 & 0,31 & $\mathbf{- 0 , 0 8}$ & 1,00 & & & & & \\
$\mathrm{Ni}$ & $\mathbf{0 , 9 8}$ & $\mathbf{0 , 9 2}$ & $\mathbf{0 , 9 8}$ & $\mathbf{0 , 9 2}$ & 0,20 & 1,00 & & & & \\
$\mathrm{Ba}$ & $\mathbf{0 , 9 4}$ & $\mathbf{0 , 9 6}$ & $\mathbf{0 , 9 6}$ & $\mathbf{0 , 9 2}$ & 0,29 & $\mathbf{0 , 9 8}$ & 1,00 & & & \\
$\mathrm{Sr}$ & $\mathbf{0 , 7 6}$ & $\mathbf{0 , 9 3}$ & $\mathbf{0 , 8 1}$ & $\mathbf{0 , 6 9}$ & 0,64 & $\mathbf{0 , 8 1}$ & $\mathbf{0 , 8 8}$ & 1,00 & & \\
$\mathrm{~V}$ & $\mathbf{0 , 9 9}$ & $\mathbf{0 , 8 9}$ & $\mathbf{0 , 9 9}$ & $\mathbf{0 , 8 8}$ & 0,22 & $\mathbf{0 , 9 8}$ & $\mathbf{0 , 9 4}$ & $\mathbf{0 , 7 8}$ & 1,00 & \\
$\mathrm{Zn}$ & $\mathbf{0 , 7 1}$ & $\mathbf{0 , 9 5}$ & $\mathbf{0 , 7 6}$ & 0,68 & 0,58 & $\mathbf{0 , 7 8}$ & $\mathbf{0 , 8 8}$ & $\mathbf{0 , 9 3}$ & $\mathbf{0 , 7 2}$ & 1,00 \\
\hline
\end{tabular}

Observando a Figura 3 (gráfico de pesos das variáveis) nota-se que a primeira componente principal explicou $81,68 \%$ da variância dos dados e a segunda componente sintetizou $13,95 \%$ da variância, perfazendo um total de $95,03 \%$ da variabilidade dos dados. Os valores estão bem representados no gráfico, onde há forte correlações entre os metais $\mathrm{Ni}, \mathrm{Cr}, \mathrm{Ba}$, Fe e V, seguido do $\mathrm{Cu}, \mathrm{Zn}$ e $\mathrm{Sr}$ que estão isolados no terceiro quadrante e também apresentam forte correlação, corroborando os resultados mostrados na correlação de Spearman.

A análise da PCA, no gráfico de pesos, também mostrou a posição do Mo no terceiro quadrante, justificando as independentes concentrações deste metal em comparação as concentrações dos outros metais estudados. Isto confirma as baixas concentrações encontradas na Tabela 3 para este metal. Além disso, a baixa associação do Mo com o Mn é também ilustrada, fato já mencionado no valor negativo do coeficiente de correlação na matriz de Spearman. 


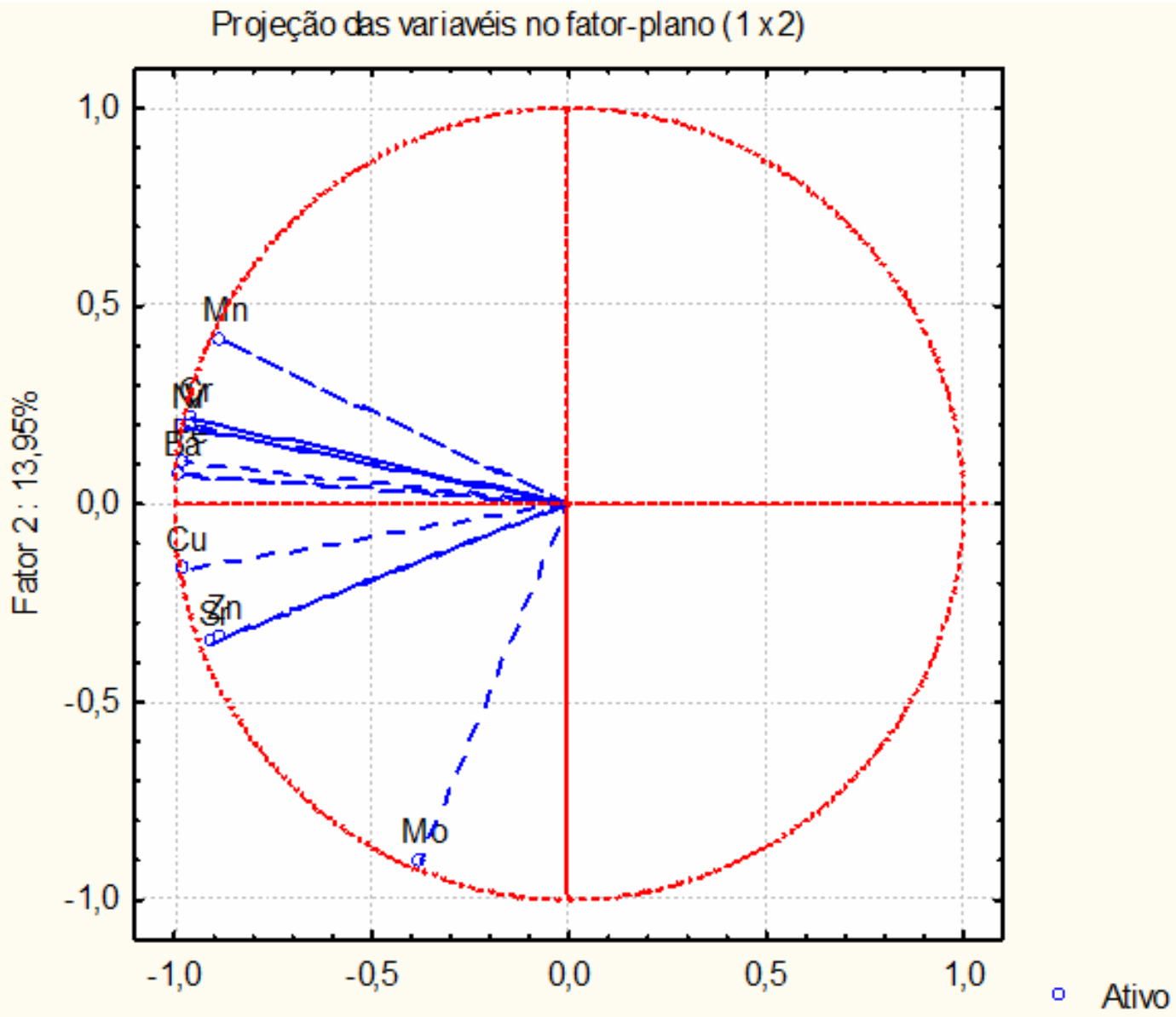

Fator $1: 81,68 \%$

FIGURA 3. Projeção das variáveis estudadas nas duas primeiras componentes principais (PC1 x PC2)

No gráfico ilustrado na Figura 4, foram produzidos quatro agrupamentos em função do local de coleta e tipo de amostragem, na qual, a primeira componente é responsável pela diferenciação entre as estações de amostras coletadas.

Observa-se que os pares de amostras 2,6 e 3, 7, em vermelho (correspondentes às amostras $P$ e $A$ das estações de coleta $S_{2}$ e $S_{3}$, respectivamente) estão melhor agrupadas, demonstrando pequena diferença entre os valores nas concentrações dos metais determinados. Por outro lado, os pares amostrais 1, 5 e 4, 8, em azul, ( $P$ e $A$ das estações $S_{1}$ e $S_{4}$ ), encontram-se agrupados, porém mais afastados. $\mathrm{Na}$ estação de amostragem $\mathrm{S}_{1}$, a influência da diferença nas concentrações de $\mathrm{Zn}$ e em menor intensidade das concentrações de $\mathrm{Mn}$ ocasionou a separação das amostras ao passo que no ponto $S_{4}$, a grande diferença nas concentrações de Mn, seguido do Zn e Sr. 


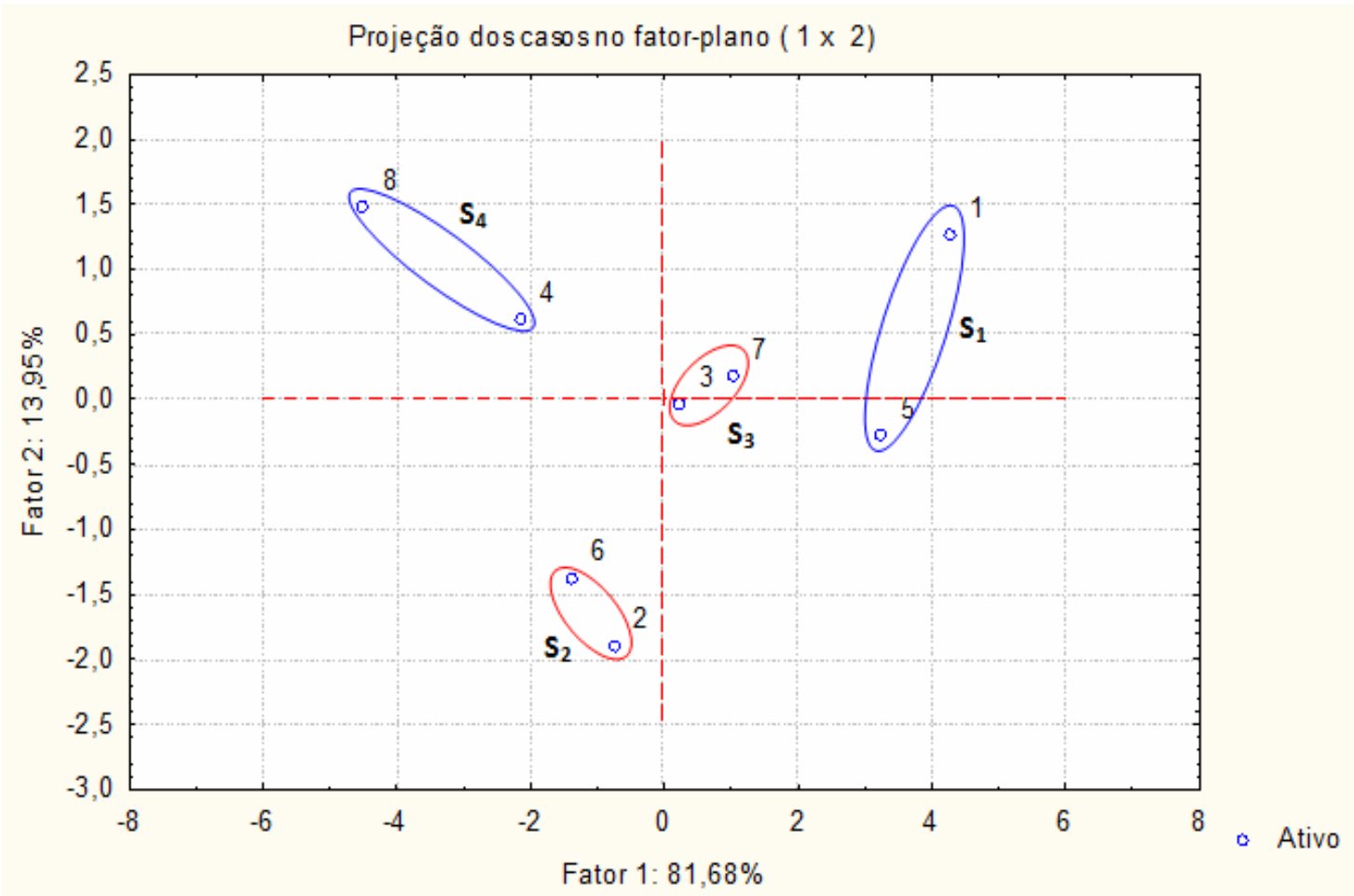

FIGURA 4. Projeção das amostras coletadas nas duas primeiras componentes principais (PC1 x PC2)

\section{CONCLUSÕES}

Os valores de concentrações obtidos para os metais $\mathrm{Cu}, \mathrm{Fe}, \mathrm{Mn}, \mathrm{Mo}, \mathrm{Ni}, \mathrm{Ba}$, $\mathrm{Sr}$ e V foram considerados não significativos. Contudo, maiores concentrações do $\mathrm{Cr}$ e principalmente de $\mathrm{Zn}$ foram encontradas nas amostras coletadas e manipuladas com material de aço inoxidável.

Através do PCA, as amostras coletadas nos pontos $S_{2}$ e $S_{3}$ indicaram um melhor agrupamento e maior separação entre as amostras dos locais $S_{1}$ e $S_{4}$, diante disso sugere-se uma maior investigação para este tipo de análise e cuidado na escolha dos processos de amostragem e tratamento das amostras numa análise química.

\section{REFERÊNCIAS}

ALMEIDA, R. A. A degradação ambiental em áreas de preservação permanente da bacia do rio Passa Vaca (Salvador-BA). Gestão de recursos hídricos. Salvador, Ingá, série Águas da Bahia, v.1, p.157-166, 2009.

CETESB; ANA. Guia Nacional de coleta e preservação de amostras: Água, Sedimento, Comunidades aquáticas e Efluentes líquidos. Brasília: DF. 2011.

FARIAS C. O,; HAMACHER C.; WAGENER A. DE L. R.; CAMPOS C DE R.; GODOY J. M.; Trace metal contamination in mangrove sediments, Guanabara Bay, Rio de Janeiro, Brazil. Journal of the Brazilian Chemical Society, Rio de Janeiro, v. 18, n. 6, p.1194-1206, 2007.

FERREIRA, S. L. C.; SILVA, L. O. B.; SANTANA, F. A. de; JUNIOR, M. M. S.; MATOS, G. D.; SANTOS, W. N. L. dos. A review of reflux systems using cold finger ENCICLOPÉDIA BIOSFERA, Centro Científico Conhecer - Goiânia, v.11 n.22; p. 3348 
for sample preparation in the determination of volatile elements. Microchemical Journal, Salvador, $2012 . \quad$ Disponível em: <http://dx.doi.org/10.1016/j.microc.2012.08.015> Acesso em: 10 nov. 2014.

LIMA, T. M. J.; TOGNELLA, M. M. P. Estrutura e função dos manguezais: revisão conceitual. Enciclopédia Biosfera, v.8, p. 1801-1827, 2012.

LUTZ, E. THOMPSON, M. I. Hot block digestion of soil, sediment, waste and tissue samples for total recoverable metals. Revision, 01 mar. 2012. Disponível em <ftp://ftp.dep.state.fl.us/pub/labs/lds/sops/5441.pdf> Acesso em: 22 abr. 2014.

NOGUEIRA, A. R. de A. Preparo de Amostras. In: VIII ENCONTRO NACIONAL SOBRE MÉTODOS DOS LABORATÓRIOS DA EMPBRAPA. Anais. Jaguariúna, São Paulo: Embrapa, 4-7 nov. $2003 . \quad$ Disponível em: $<$ http://www.cnpsa.embrapa.br/met/images/arquivos/08MET/Palestras/preparoamost ras.pdf> Acesso em: 22 nov. 2014.

SERTEK, J. P. Estudos das contaminações provenientes do processo de cominuição de amostras geológicas. Dissertação (Mestrado em Petrologia Ígnea e Metamórfica) - Instituto de Geociências, Universidade de São Paulo, São Paulo, 2010.

SILVA, M. A.; BATISTA, R. J. R.; ROCHA, T. R.; AMARANTE, C. B.; FALCÃO, E. H. $O$. Teor de macronutrientes em sedimentos de manguezais: ilha de llarana e Cuiarana - Pará - Brasil. Enciclopédia Biosfera, v.9, p. 2014-2028, 2013.

TEMPLETON, D. M.; ARIESE, F.; CORNELIS, R.; DANIELSSON, L.G., MUNTAU, H., LEEUWEN, H.P., LOBINSKI, R. IUPAC. Guidelines for terms related to chemical speciation and fraction of elements. Definitions, structural aspects and methodological approaches. Pure Applied Chemistry, v. 72, p.1453-14470, 2000.

TEÓFILO, R. F.; FERREIRA, M. M. C. Quimiometria II: Planilhas eletrônicas para cálculos de planejamentos experimentais, um tutorial. Química Nova, Campinas, v. 29, n.2, p. 338-350, 2006.

THOMPSON, G.; BANKSTON, D. C. Sample Contamination from Grinding and Sieving Determined by Emission Spectrometry. Applied Spectroscopy, Massachusetts, v. 24, n. 2, p. 210-219, 1970 